\title{
Development of a reconfigurable pallet system for a robotic cell
}

\author{
Martin Koekemoer ${ }^{1}$, and Igor Gorlach ${ }^{1}$ \\ ${ }^{1}$ Nelson Mandela University, North Campus, Department of Mechatronics, Port Elizabeth, South Africa
}

\begin{abstract}
Advanced manufacturing systems allow rapid changes of production processes by means of reconfigurability providing mass customisation of products with high productivity, quality and low costs. Reconfigurable Manufacturing Systems (RMS) employ conventional as well as special purpose CNC machines, robots and material handling systems. In customised automated assembly, a number of different workpieces need to be processed simultaneously at various workstations according to their process plans. Therefore, a material handling system is an important part of RMS, whose main task is to provide reliable, accurate and efficient transfer of materials according to the process scheduling, without bottlenecks and stoppages. In this research, a reconfigurable pallet system was developed to facilitate automated robotic assembly for a highly customised production environment. The aim is to design a material handling system for conveying, sorting and processing of parts, which are supplied by robots and part feeders in different configurations. The developed pallet system provides a low-cost solution and it includes four flexible conveyors and part handling devices. All the elements of the system were successfully integrated with an intelligent controller. A user-friendly human machine interface provides easy reconfigurability of the pallet system and interfacing with robots, processing stations and part feeding sub-systems. The main advantages of the developed material handling system are the ease of operation, its reconfigurability and low-cost. The system demonstrates the advantages of reconfigurable material handling systems and it can be employed for training purposes.
\end{abstract}

\section{Introduction}

Reconfigurable Manufacturing Systems (RMS) evolved as a type of Flexible Manufacturing Systems (FMS), which have the following characteristics: modularity, integrability, customisation, convertibility and diagnosability. RMS are intended for mass customisation by means of rapid changeover of machines, production processes and the whole system logistics.

Research focuses in RMS include developing of systems [1-4], specialised machines, [5-7], process planning [8], fixtures and sub-systems [9]. Holonic architecture of modular control of reconfigurable conveyor system was reported in [10-11]. A modelbased approach in designing of reconfigurable conveyor systems presented in [12] evaluates the context of conveyor topology for both the physical and cyber issues. An object oriented design and analysis in designing of reconfigurable conveyors is reported in [13].

Modern automated assembly is a multi-stage process, performed by robots and machines, which requires a variety of workpiece to be transferred through the stations by a material handling system quickly, accurately and reliably. This goal can be achieved by developing an intelligent pallet handling system. Firstly, a workpiece holding platform needs to be chosen. Pallets, for example, can provide a stable surface on which assembly can be performed and improve the customisation capabilities of the system as they can be fitted with reconfigurable fixtures to hold different workpieces in place. By being able to identify a pallet uniquely, multiple products of a similar nature can be assembled using the same system. The use of pallets also improves traceability of processes. For example, if a company produces electronic circuit boards of type A and type $\mathrm{B}$, which contain similar components but are dimensionally varying - pallets with unique fixtures can be used to hold each base. By identifying the pallet, the machine can choose components to place appropriately to fit the needs of type A or B - this links to customization. Once a pallet has been processed by a station, its status is updated to reflect at which stage of the assembly process the workpiece is.

Secondly, a material handling system must be reconfigurable in order to provide for modifications of equipment and changes of layouts as required. This requirement is achieved by using the principles of modularity and scalability in designing all sub-systems of a conveyor system, such as: mechanical, electrical, control and communication. 
Thirdly, the design of a reconfigurable material handling system needs to be according to the principles of integrability and diagnosability, which are achieved through developing appropriate physical and software interfaces for seamless integration with robots, assembly machines and their controllers. These three criteria are addressed in the design process described below.

\section{Reconfigurable pallet system design}

\subsection{System Architecture Design}

The proposed robotic cell was designed for multi-stage assembly of different products supplied by the reconfigurable pallet system. The robotic cell layout can accommodate a number of robots, processing and buffer stations, which can be easily reconfigured as required. This requirement is achieved because the pallet system satisfies all the three above-mentioned criteria.

The first criterion was addressed by selecting flexible controllable conveyors with traceable pallets and adjustable workstations. The identical conveyors have special mechanical and electrical interfaces to latch them in a cell. A number of workstations or buffers and their positions on conveyors can be altered as they have modular design features. This is important for facilitating assembly and processing operations with buffer stations between them. A basic robotic cell layout requires at least four conveyors. The development of a cell layout is achieved with a software program compiled using Visual Studio C\#, which allows a user to configure a cell by adding robots and process machines, select a required number of work and buffer stations, decide on a material flow process and assign the stations to the operations.

The software program has a number of GUI to facilitate the design process. The 'Layout design' GUI is used for a development of the cell layout, which can be saved and recalled when needed, Fig. 1. The 'Process' GUI allows the user to select the Robot Service Request (RSR) according to the process plan and workstation pallet servicing. An image of a saved layout is displayed in the 'Process' GUI such that the configurator is aware where workstations and buffer stations were placed. Once the process 'recipe' is complete monitoring and control could occur using the 'Monitoring and Control' GUI, which provides stop, start and E-Stop functionality while displaying the state of all sub-systems, buffer stations, workstations, drives etc. Fig. 1 shows the GUI of the robotic cell layout chosen in this research. The robotic cell consists of a Mitsubishi RV-2AJ robot, four flexible conveyors and eight stations. This layout can accommodate up to four robots and/or processing machines along the conveyors. Pallets can travel in both directions if required by the material handling process. The cell layout can be easily altered, for example, the conveyors can be arranged in an open loop or in a continuous line. By adding conveyors and creating branches, the pallet system can have many configurations and, therefore, the second criterion of the design is achieved.
In the current configuration, Conveyors 1 and 4 both have two buffer stations, while Conveyors 2 and 3 both have one workstation and one buffer station. The Mitsubishi RV-2AJ Robot is used for assembly and loading/unloading tasks.

The operation sequence is defined as follows: pallets 1, 2, 3 and 4 travel along the closed loop of four
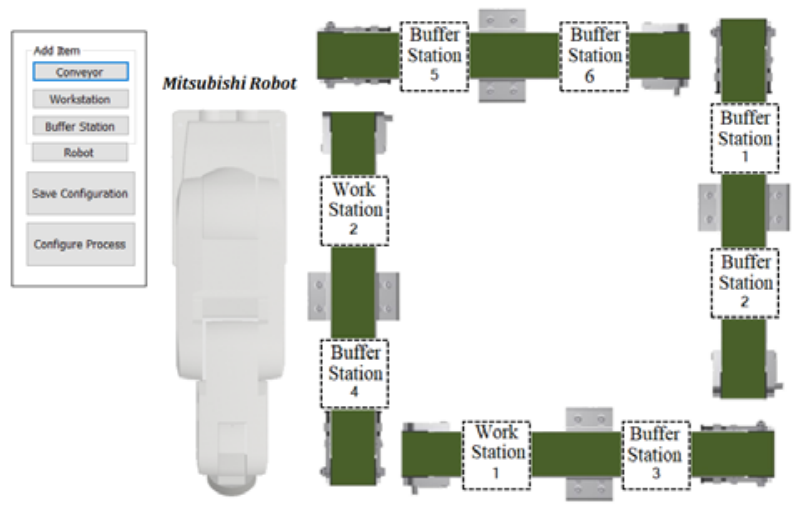

conveyors. Workstation 1 services pallets 1 and 3 while workstation 2 services pallets 2 and 4 . This operation sequence is then executed through SCADA by the cell Master controller. The user can visualise the status of each station and control the entire process via HMI.

Fig. 1. GUI of the model of the pallet system

\subsection{Conveyor and Workstation Designs}

A modular design approach was applied in the development of the reconfigurable pallet system and all its elements. Flexible conveyors were designed to convey pallets in continuous or indexing motion in both directions, Fig 2. The overall mechanical design of a conveyor is such that it would allow it to be directly attached to other conveyors at the ends or on sides, which would provide a flow of material in many configurations.

The flexible conveyor is driven by a $12 \mathrm{~V} 1 \mathrm{~A}$ stepper motor using a PWM signal from a microcontroller via a stepper motor driver. All four conveyor stepper motors are controlled by one microcontroller, which in turn is controlled by the cell controller on the higher level.

The work/buffer station was designed as a completely standalone unit, which incorporates a means of stopping and identifying of pallets, and, therefore, it includes a stopper and RFID reader, shown in Fig. 2, as well as a pallet lifting mechanism, shown in Fig. 3. The pallet stopper mechanism is driven by a micro servo motor while the pallet lifting mechanism employs electrically driven cams, with each servo's position controlled by a pulse signal sent from a microcontroller. 


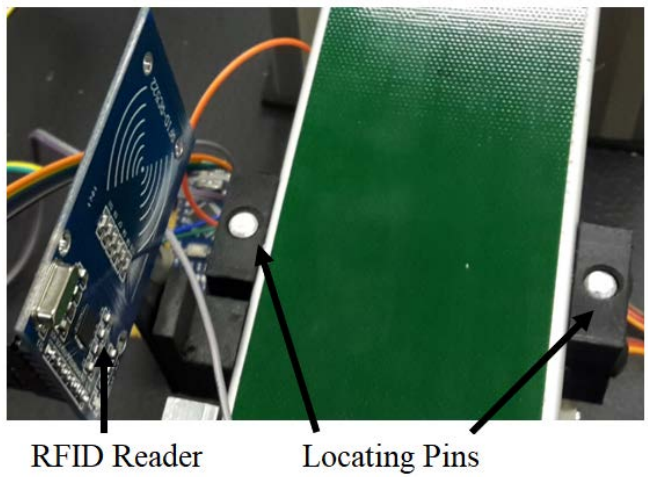

Fig. 2. The pallet locating mechanism

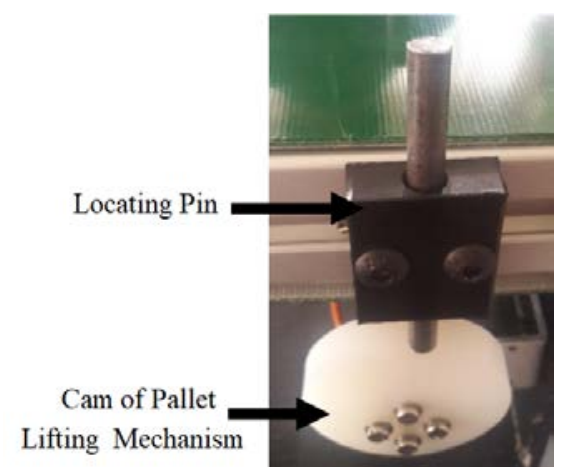

Fig. 3. The pallet lifting mechanism

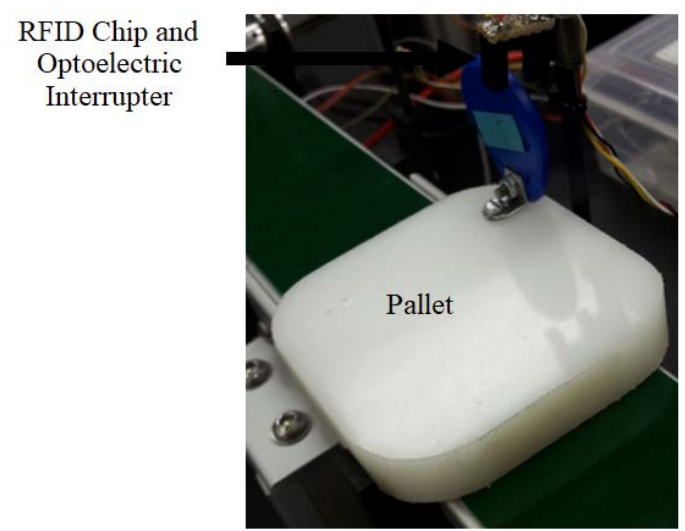

Fig. 4. The pallet with the RFID chip

All pallets are uniquely identified with an integer value stored on a RFID chip attached to the pallet, Fig.4. When a pallet enters a workstation its number is read from the RFID chip using a MFRC522 RFID reader, which communicates through a Serial Peripheral Interrupt (SPI) interface to the station controller. Depending on the process flow, the pallet either stopped at the workstation or it is allowed to pass through to the next station. The presence of the pallet in a station is determined using optoelectric interrupters.

All the control functions of a station are executed by a microcontroller, which communicates with the cell controller, whose functions are described below.

\subsection{Control system}

All controllers pertaining to the reconfigurable pallet system are realised on the Arduino Nano platform, programmable in $\mathrm{C}++$. The platform offers the use of standard libraries for common components. The Arduino Nano controllers meet all the requirements of reconfigurability as they can be easily interfaced with various types of controllers and networks. In addition, they are a compact as well as a low-cost solution.

The control system has the distributed architecture, Fig. 5. The Host of the control system runs the SCADA program on a PC which communicates with the robot and cell-controller, for the pallet system, via serial interface. Through the SCADA program a user can control and monitor the assembly and material-handling processes as well as the status of the robot, conveyors, buffers and workstations in real time.

The cell controller for the pallet system uses an InterIntegrated Circuit (I2C) interface to communicate with all sub-controllers. These consist of workstation controllers and a conveyor controller. The workstation controllers are responsible for the eight stations while the conveyor controller is responsible material-handling, in the form of multiple belt conveyors. The I2C interface provides a means for data receiving and transmission, this allows the cell controller to send commands to the sub-controllers and receive the state of sub-controllers in real time. In turn the sub-controllers use digital $\mathrm{I} / \mathrm{O}$ to interface with sensors, actuators and external assembly equipment.

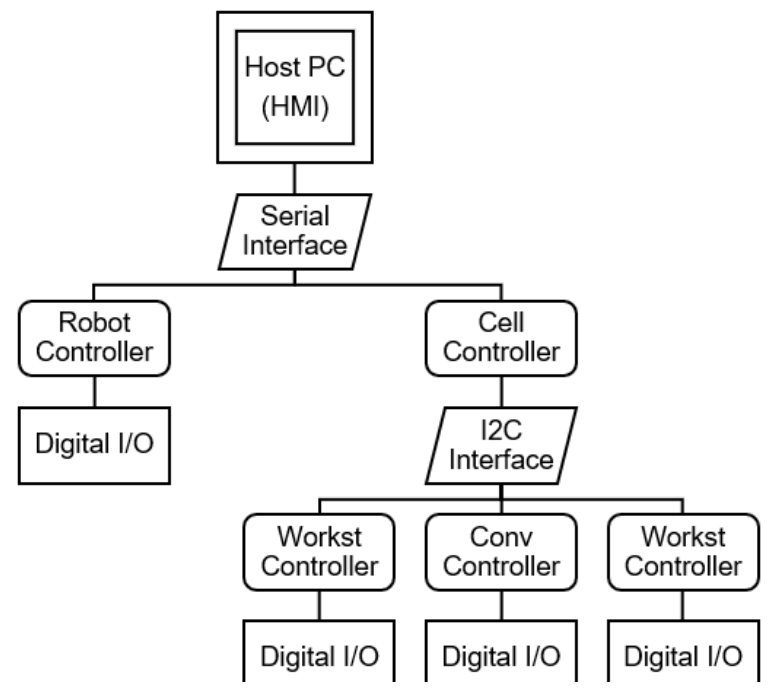

Fig. 5 Schematic diagram of the control system

The 'Monitoring and Control' screen, shown in Fig. 6, was developed as the main HMI as part of the SCADA program. The monitoring functions provide all the required information regarding the state of each workstation, buffer station and conveyor motor drive. The data is requested by the cell controller using a periodic interrupt sent to each sub-controller. The data exchange takes place through $\mathrm{I} 2 \mathrm{C}$ in a master readerslave writer configuration. The state of each subsystem is then relayed via the cell controller to the host PC which are interfaced via a micro Universal Serial Bus (USB) connection. 
The control functions provide a means of controlling the subsystems via the cell controller. The system can be started and stopped remotely via the HMI as shown in Fig. 6. These commands are sent from the host PC to the cell controller via serial communication. The cell controller then relays the command to each subcontroller via the $\mathrm{I} 2 \mathrm{C}$ interface in a master writer-slave reader configuration.

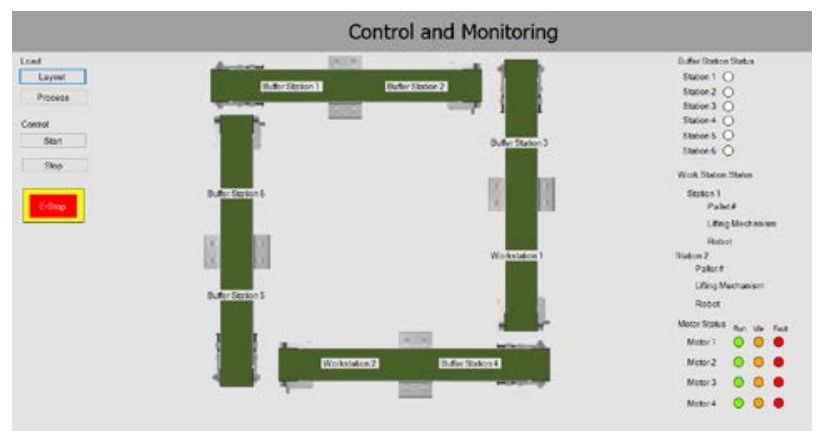

Fig 6. HMI Interface controller

To run the pallet system a layout and process needs to be selected. This is achieved by using the appropriate open commands on the 'Monitoring and Control' screen. After the layout and process is selected the user can activate and control the system from the Host PC using the HMI.

The control logic of the workstation program is shown in Fig. 7. Each workstation is configured with a 'Pallet to Service' list and is granted a unique ID allowing it to be identified by the cell controller. Each workstation can interface with external assembly equipment such as a robot. Hence each workstation can have its own dedicated assembly equipment, or the stations can work with a single assembly device as it appears in this case. Appropriate programs relating to the current system configuration need to be loaded onto external assembly equipment as required.

An assembly program was created for the Mitsubishi RV-2AJ robot using COSIMIR simulation software. Robots and processing machines typically provide digital $\mathrm{I} / \mathrm{O}$ for communication with other devices and to allow for real time control. Therefore, the transmission of signals between the robot and each workstation controller, takes place via hardwired signals.

The workstation controller sends a signal via digital $\mathrm{I} / \mathrm{O}$ to the robot controller once the correct pallet is in station to commence a task or RSR. The robot controller calls and executes the required program. After completion of the tasks outlined in the program, the robot controller sends a signal to the workstation controller that requested the task to indicate that that the work is complete. As the robot digital $\mathrm{I} / \mathrm{O}$ logic level is $24 \mathrm{~V}$ and the Arduino Nano logic level is $5 \mathrm{~V}$, signal conditioning is applied to facilitate communication between the devices. $24 \mathrm{~V}$ signals are sent from the Arduino to the robot controller by activating a relay with a $5 \mathrm{~V}$ logic level and providing $24 \mathrm{~V}$ output. The $24 \mathrm{~V}$ signal sent from the robot controller to the Arduino is stepped down to $5 \mathrm{~V}$ using a voltage regulator IC. Robot signals are monitored on the 'I/O Monitor' of the
COSIMIR simulation software, which is used for offline programming and online monitoring of robots.

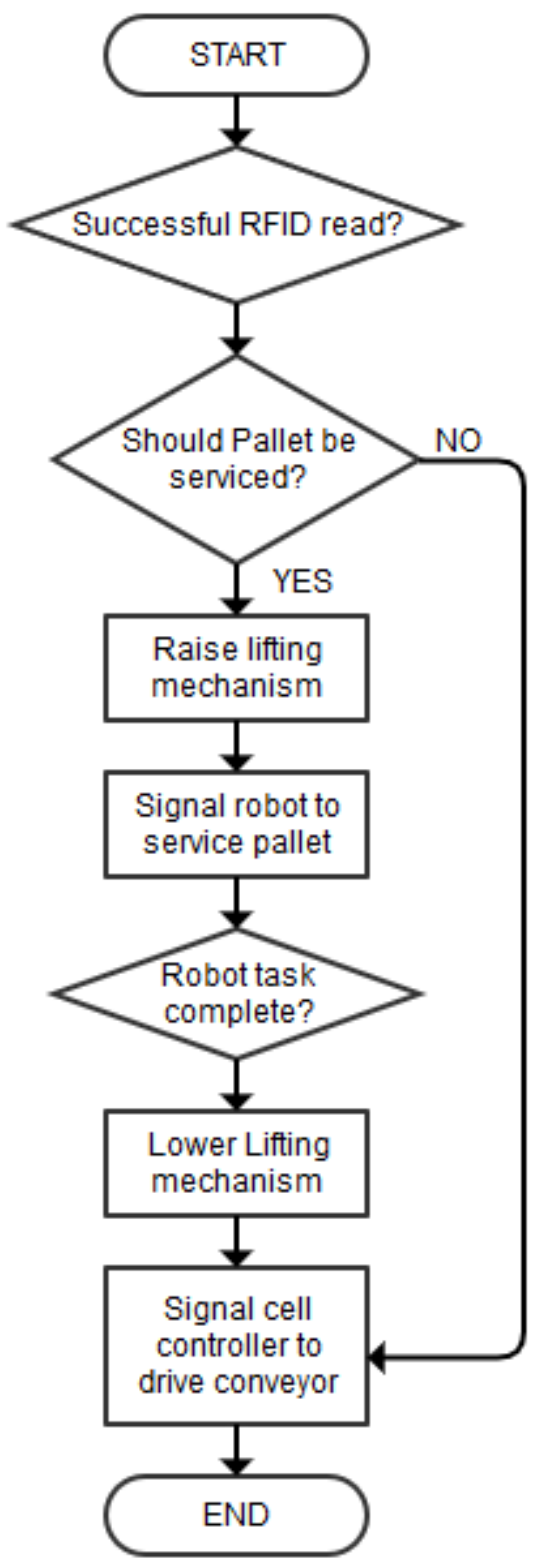

Fig 7. Flow diagram of the workstation control program

\section{Results and Analysis}

The developed robotic cell with the reconfigurable pallet system and the Mitsubishi RV-2AJ robot is shown in Fig.8. Four pallets with different components were transported by the conveyors through eight workstations and buffers according to the process plan.

The pallets were monitored and stopped as required and the robot loaded/unloaded the parts. 


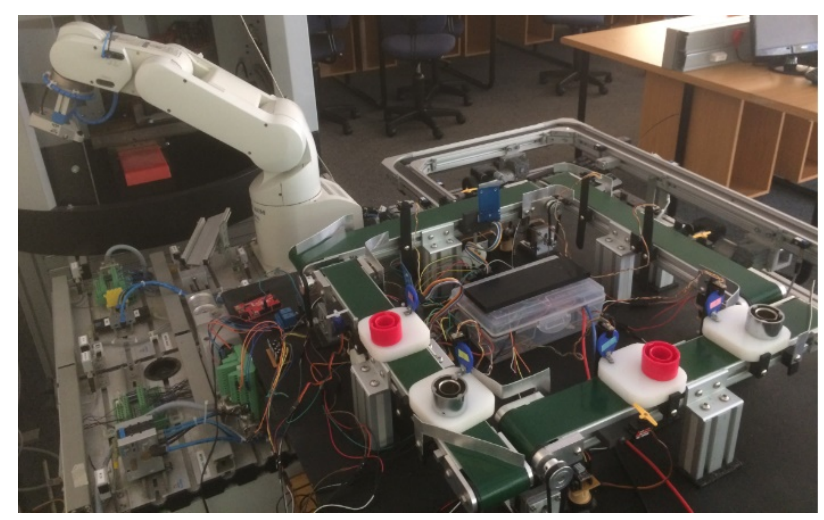

Fig 8. Robotic cell with the reconfigurable pallet system

A number of performance measures were evaluated during the testing phase, which include capability, reliability, accuracy and repeatability. The conveyor is capable of delivering a load of $2 \mathrm{~kg}$ with variable speed from 0.02 to $0.1 \mathrm{~m} / \mathrm{s}$. Workstations 1 and 2 are considered as critical locations for positional accuracy. This was achieved by means of locating pins, which engaged in locating holes of pallets ensuring a positional accuracy in the $\mathrm{X}$ and $\mathrm{Y}$ direction for each pallet entering a workstation. Workstation 1 was able to accurately position pallets 1 and 3 in the range of $\pm 1 \mathrm{~mm}$ with a repeatability of above $95 \%$. Similar results were obtained for Workstation 2 servicing pallets 2 and 4.

The positional accuracy of the stepper motors was verified by sending 200 pulses to the stepper motor. Since the resolution of the stepper motor was 1.8 degrees per pulse, 200 pulses would constitute a full 360 degrees rotation. Hence by determining if the motor made a full rotation in 200 pulses the positional accuracy and variable speed control could be verified. A straight vertical line was drawn on the motor pulley, after 200 pulses; for every test, the angle between the line and a flat horizontal surface was measured in order to obtain the degrees rotated. The same procedure was carried out for the driven pulley, which rotated 2.5 times for each single rotation of the motor pulley based on the ratios of the pulleys' number of teeth.

The cell sub-controllers and motor drivers were tested by measuring pulses sent to the stepper driver from the microcontroller using an oscilloscope. For a range of pulse frequencies sent by the same pin on the microcontroller, the signal received by the stepper driver was measured. A pulse duration corresponded to a delay in milliseconds in Arduino pulsing code. Hence, a delay of 10 milliseconds corresponded to a pulse with a 10 millisecond high and a 10 millisecond low portion, which corresponds to a frequency of $50 \mathrm{~Hz}$. The test was repeated three times for pulses of duration 10 milliseconds and 20 milliseconds.

The quality of communication between the microcontrollers using $\mathrm{I} 2 \mathrm{C}$ communication was evaluated by sending bits at a frequency of $50 \mathrm{~Hz}$ and assessing the message integrity, which was found to be satisfactory. The same approach was used to test serial communication between the Host PC and the Arduino.
Overall, the system was able to service 1500 pallets without any failures, demonstrating high reliability for a prototype.

\section{Conclusion}

In this research, a reconfigurable pallet system for a robotic cell for assembly and processing was developed, which includes the 5-DOF Mitsubishi robot, four flexible conveyors forming a closed loop, part handling devices and the control system. The cell is designed to facilitate automated robotic assembly for a highly customised production environment. This is achieved by reconfiguring the conveyor-pallet system according to the required robotic cell architecture and corresponding assembly processes. The development process is facilitated by a simulation software program, which allows a user to create the system model, which is then linked with the control system, which in turn communicates to the cell controllers via SCADA. Realtime control and monitoring is provided by interactive HMI. The main advantages of the developed system are the ease of operation, its reconfigurability and low-cost. By applying appropriate hardware and software modifications the Arduino Nano controllers can connect to a Wi-Fi network. This means that each workstation controller, the conveyor controller and cell controller for the pallet system could exist as separate entities connected through a wireless network. Future work could focus on creating such a cyber-physical system in which devices can communicate through the internet of things (IoT). By allowing this, the system can tap into benefits offered by Industry 4.0 such as improved efficiency, improved customisation ability, improved agility and lower cost.

\section{Acknowledgments}

The authors would like to acknowledge the support and financial assistance provided by Isuzu Motors South Africa.

\section{References}

[1] Y. Koren \& A.G. Ulsoy, Reconfigurable Manufacturing Systems, Engineering Research Center for Reconfigurable Machining Systems (ERC/RMS), The University of Michigan, Ann Arbor, 1997.

[2] M.G. Mehrabi \& A.G. Ulsoy, State-of-the-Art in Reconfigurable Manufacturing Systems, Report \#2, Vol. I, Engineering Research Centre for Reconfigurable Machining Systems (ERC/RMS), University of Michigan, Ann Arbor, 1997.

[3] M.G. Mehrabi \& A.G. Ulsoy, State-of-the-Art in Reconfigurable Manufacturing Systems, Report \#2, Vol. II, Engineering Research Centre for Reconfigurable Machining Systems (ERC/RMS), University of Michigan, Ann Arbor, 1997. 
[4] M.G. Mehrabi, A.G. Ulsoy \& Y. Koren, Reconfigurable Manufacturing Systems: Key Feature Manufacturing, Journal of Intelligent Manufacturing, 11/4, 2000, 403-419.

[5] R. Katz \& Y. Moon, Virtual Arch Type Reconfigurable Machine Tool Design: Principles and Methodology, Department of Mechanical Engineering, The University of Michigan, Ann Arbor, 2000.

[6] M. Simpson \& I.A. Gorlach, Development of a Reconfigurable Machine Tool. Proc. Conf. on Competitive Manufacturing, COMA'10, Stellenbosch, South Africa, 2010.

[7] E. Murena, K. Mpofu, J. Trimble, Framework for Developing a Web based Process Plan for Reconfigurable Press Brake Bending Machine, Proc. Conf. on Competitive Manufacturing, COMA'16, Stellenbosch, South Africa, 2016.

[8] A. Hees, L. Lipp, S Braunreuther, G Reinhart, System for Production Planning of Reconfigurable Manufacturing Systems, Proc. Conf. on Competitive Manufacturing, COMA'16, Stellenbosch, South Africa, 2016.

[9] E. Slabbert, Low Level Task Execution, Programming and Control for Jigs, Fixture and Equipment, Proc. Conf. on Competitive Manufacturing, COMA'16, Stellenbosch, South Africa, 2016.

[10] D.M. Masendeke, A.H Basson, Communication in a Labview Based Holonic controller, Proc. Conf. on Competitive Manufacturing, COMA'16, Stellenbosch, South Africa, 2016.

[11] M.J. Kotze, Modular Control of a Reconfigurable Conveyer System, PhD, Stellenbosch University, South Africa, 2016.

[12] K. An, A, Trewyn, A. Gokhale, S. Sastry, ModelDriven Performance of Analysis of Reconfigurable Conveyor Systems used Material Handling Applications, Proceedings of the 2011 IEEE/ACM Second International Conference on CyberPhysical Systems, 2011, pp 141-150.

[13] J.K.L. Ho, P.G. Ranky, Object oriented modelling and design of reconfigurable conveyors in flexible assembly systems. International Journal of Computer Integrated Manufacturing, Vol. 10, Issue 5, 1997, pp 360-379. 